\title{
HIV-1 Gag-Virus-Like Particles Induce Natural Killer Cell Immune Responses via Activation and Maturation of Dendritic Cells
}

\author{
Myint Oo Chang ${ }^{a}$ Tomoyuki Suzuki $^{b}$ Hitoshi Suzuki $^{b}$ Hiroshi Takaku $^{a}$ b \\ ${ }^{a}$ High Technology Research Centre, and ${ }^{b}$ Department of Life and Environmental Sciences, Chiba Institute of \\ Technology, Narashino, Japan
}

\section{Key Words}

Gag-virus-like particles • Dendritic cells • Natural killer cells • HIV - Innate immunity

\begin{abstract}
Despite the extensive efforts that have been made to combat acquired immune deficiency syndrome (AIDS), the number of people infected each year with human immunodeficiency virus type 1 (HIV-1) is still increasing worldwide, and a safe and effective vaccine to control HIV infection is urgently needed. Recently, the natural killer (NK) cell-mediated innate immune response, which represents the first line of defense against infections, has attracted attention for its role in combating HIV infection and disease progression. In the present study, we investigated the immunogenic ability of HIV-1 Gag-virus-like particles (Gag-VLPs) to induce NK cell immune responses in vitro and in vivo. Gag-VLPs efficiently activated human monocyte-derived dendritic cells (MDDCs), eliciting MDDC maturation with an associated increase in the surface expression of CD80, CD86 and MHC classes I and II, MDDC proliferation and proinflammatory cytokine production. Gag-VLP-treated MDDCs subsequently activated autologous NK cells, leading to their proliferation and production of interferon- $\gamma$ and to the upregulation of NK cell cytotoxicity against YAC-1 cells and HIV-1-infected $\mathrm{CD} 4^{+} \mathrm{T}$ cells. In addition, we introduced a 2-phase immunization strategy in BALB/c mice to assess the role of DCs in the induction of NK cell immune responses by Gag-VLPs in vivo.
\end{abstract}

\section{KARGER}

Fax +41613061234 E-Mail karger@karger.ch www.karger.com
(C) 2012 S. Karger AG, Basel

$1662-811 X / 12 / 0042-0187 \$ 38.00 / 0$

Accessible online at:

www.karger.com/jin
Our findings reveal that Gag-VLPs efficiently activate DCs, which in turn induce innate and Gag-specific immune responses in NK cells.

Copyright $\odot 2012$ S. Karger AG, Basel

\section{Introduction}

Control of human immunodeficiency virus (HIV) infection by highly active antiretroviral therapy has greatly decreased morbidity and mortality due to acquired immune deficiency syndrome (AIDS). However, despite enormous efforts, the number of HIV-1-infected individuals increases on a daily basis, and we still lack prophylactic and therapeutic vaccines against HIV-1. Several vaccine components such as live attenuated viruses, recombinant viral vectors and DNA vaccines have been proven to be effective in inducing cellular and humoral immune responses; however, serious safety concerns and the limited efficacy of some of these vaccine components prevent their clinical application as anti-HIV vaccines. The possible risks of live attenuated viruses and recombinant viral vectors, including (1) potential reversion to a virulent pathogenic form of the attenuated virus, especially in immunodeficient recipients, (2) possible recombination of the vaccine strain with wild-type pathogenic

Co-corresponding author:

Dr. Myint Oo Chang

E-Mail c_myintoo@hotmail.com

Prof. Hiroshi Takaku

Department of Life and Environmental Sciences, Chiba Institute of Technology

2-17-1, Tsudanuma, Narashino

Chiba 275-0016 (Japan)

Tel. +81 47478 0407, Fax +81 47471 8764, E-Mail hiroshi_takaku@ it-chiba.ac.jp 
virus in an infected individual, (3) dysregulation of the immune system by viral proteins and (4) ability of the proviral genome to integrate into the host genome, have prevented their approval for use in humans $[1,2]$. Furthermore, several potential risks of DNA vaccines, including tumor induction due to chromosomal integration, autoimmune reactions as a result of the induction of anti-DNA antibodies or adverse reactions due to the biological activity of the vector or the expressed antigens have not yet been ruled out [3]. Because of these difficulties, a safe and effective HIV vaccine suitable for prophylactic and therapeutic use in humans is urgently needed.

Virus-like particles (VLPs) are stable, self-assembling, nonreplicating and noninfectious particles capable of inducing immune responses without containing any viral genome or other potentially toxic viral gene products [46]. Recent research in HIV vaccine strategies has focused on the use of VLPs as highly attractive HIV-1 vaccine candidates because of their ability to elicit humoral and cellular immunity more effectively than soluble proteins $[7,8]$.

VLPs are incorporated into dendritic cells (DCs) via actin-dependent macropinocytosis and endocytosis. VLP uptake is also induced by receptor-mediated endocytosis. Mannose-recognizing receptors, including the DC-SIGN receptor, which belongs to the $\mathrm{C}$-type lectin family, are reportedly involved in this process. The DC-SIGN receptor, present on the DC surface, recognizes VLPs and enhances internalization for lysosomal degradation, antigen processing and cross-presentation on both MHC class I (MHC I) and MHC class II (MHC II) molecules. VLPs induce maturation and activation of monocyte-derived DCs (MDDCs), and this effect is partially mediated through Toll-like receptors [9-11]. Previous reports have shown that Gag-VLPs induce $\mathrm{CD} 4^{+} \mathrm{T}$ cell activation and $\mathrm{CD}^{+}$cytotoxic T lymphocyte (CTL) responses as well as B cell-mediated humoral immunity [9, 12-17]. Despite these findings, little is known about the ability of GagVLPs to elicit natural killer (NK) cell immune responses.

DCs are the most potent antigen-presenting cells of the immune system and play a pivotal role in the initiation and regulation of immune responses to various antigens. Antigen-loaded DCs acquire a mature phenotype associated with the production of proinflammatory cytokines and stimulate $\mathrm{CD} 4^{+} \mathrm{T}$ cells and CTL responses [18-20]. The mature DCs migrate towards the lymphoid organs, where they interact with NK cells, inducing the activation and proliferation of these cells. The interaction between DCs and NK cells initiates innate signals that orchestrate subsequent adaptive immune responses [21-25].
NK cells are the primary effector cells of the innate immune system and are essential for the immune control of infections and tumors through production of cytokines such as interferon (IFN)- $\gamma$ and the potent lysis of infected cells or tumor cells, without the need for prestimulation. Notably, other than activated T cells, NK cells are the only known source of IFN- $\gamma$ which shapes the pattern of innate and adaptive immune responses [26-28]. The innate immune response of NK cells against HIV is crucial both for the subsequent course of the infection and for the induction of an efficient adaptive response. Both NK cell cytotoxicity and cytokine production are impaired in HIV-1 viremic patients. Preservation of NK cell activity and cell number correlates with lower plasma viral load and slower progression to AIDS [29, 30]. Moreover, because NK cell responses following vaccination correlate with clinical outcome more closely than do $\mathrm{T}$ cell responses, the effectiveness of an HIV vaccine should be evaluated by monitoring the potentially important NK cell responses after immunization [31].

In this article, we demonstrate that Gag-VLP-treated MDDCs (VLP-DCs) underwent activation and maturation characterized by the increased surface expression of costimulatory molecules, including CD80, CD86, MHC I and MHC II, and the production of proinflammatory cytokines, including IL-12 p70, IL-15, TNF- $\alpha$, IFN- $\alpha$ and IFN- $\gamma$. MDDCs subsequently activated NK cells, inducing their proliferation, release of IFN- $\gamma$ and enhanced $c y-$ totoxicity. Next, we immunized BALB/c mice with GagVLPs in a phase I immunization study to examine the activation of DCs and NK cells in vivo. These experiments were followed by a phase II immunization study in which mice were inoculated with splenic DCs of phase Iimmunized mice to assess the contribution of DCs to the induction of $\mathrm{NK}$ cell immune responses.

In conclusion, we show that Gag-VLPs induce activation and maturation of DCs, which then mediate NK cell innate and Gag-specific immune responses. Our findings reveal that Gag-VLPs are an effective HIV-1 vaccine candidate for prophylactic and therapeutic purposes in humans.

\section{Materials and Methods}

Mice and Cell Culture

Six- to 8-week-old female BALB/c mice were purchased from Nippon SLC (Hamamatsu, Japan). All mice were maintained under specific-pathogen-free conditions. All animal experiments were carried out in compliance with institutional guidelines and approved by the animal experimentation committee of the Chiba 
Institute of Technology. Spodoptera frugiperda (Sf9) insect cells were grown at $27^{\circ} \mathrm{C}$ in $\mathrm{BD}$ Gold serum-free medium containing $100 \mu \mathrm{g} / \mathrm{ml}$ kanamycin sulfate. HeLa cells and HEK-293T cells were maintained in DMEM culture medium (Sigma), and YAC-1 cells were maintained in RPMI-1640 culture medium (Sigma). Both DMEM and RPMI-1640 were supplemented with 10\% fetal bovine serum (Life Technologies), $100 \mathrm{U} / \mathrm{ml}$ penicillin and 100 $\mu \mathrm{g} / \mathrm{ml}$ streptomycin (Sigma).

Generation of Recombinant Baculovirus and Wild-Type HIV-1 The baculovirus transfer vector pAcCAG-gag containing the HIV-1 gag gene was generated by cloning of cDNA encoding the HIV-1 (NL4-3) gag gene into the multiple cloning site of the baculovirus transfer vector pAcCAG-MCS2, under control of the mammalian CAG promoter. HIV-1 gag was first cloned into the DraIII and NruI sites of pcDNA3.1 and then excised with KpnI and NotI endonucleases and ligated into the KpnI and NotI sites of the pAcCAG-MCS2 vector. Recombinant baculovirus (AcCAG-gag) was generated by cotransfection of Sf9 cells with the baculovirus transfer vector $\mathrm{pAcCAG-gag}$ and AcMNPV DNA using a $\mathrm{BD}$ Gold Baculovirus transfection kit (BD Biosciences) according to the manufacturer's protocol. AcCAG-gag was expanded in Sf 9 cells, and the titer was determined by plaque assay. Wildtype HIV-1 NL4-3 (X4-tropic) was generated by transfection of HEK-293T cells with HIV-1 plasmid (pNL4-3) using FuGENE 6, and culture supernatant was collected on day 2. Viral p24 content was measured by ELISA using Lumipulse.

\section{Production of Gag-VLPs in HeLa Cells}

HeLa cells were infected with recombinant baculovirus AcCAG-gag at a multiplicity of infection of 100 for $1 \mathrm{~h}$ at $37^{\circ} \mathrm{C}$, washed twice with PBS to thoroughly remove the baculovirus and then replenished with fresh culture medium. After incubation for 3 days, the culture supernatant was clarified by centrifugation at $2,500 \mathrm{rpm}$ for $20 \mathrm{~min}$ and filtered through a $0.45-\mu \mathrm{m}$ filter. VLPs were then pelleted at $19,000 \mathrm{rpm}$ for $2 \mathrm{~h}$ at $4^{\circ} \mathrm{C}$ with a Beckman NVT-100 rotor, resuspended in PBS, purified by $20-60 \%$ sucrose gradient centrifugation at 25,000 rpm for $90 \mathrm{~min}$ and again resuspended in PBS. Protein concentrations of purified Gag-VLP samples were determined using a $\mathrm{BCA}^{\mathrm{TM}}$ protein assay kit (Pierce). Gag-VLP preparations were determined to be free of endotoxin $(<0.01$ endotoxin units $/ \mathrm{ml})$ using a Pyrodick endotoxin kit (Seikagaku Co., Tokyo, Japan).

\section{Preparation of Human MDDCs}

Human peripheral blood mononuclear cells were separated from buffy coats using Ficoll-Paque density gradient centrifugation. Monocytes were allowed to adhere to plastic plates at $37^{\circ} \mathrm{C}$ for $2 \mathrm{~h}$, and nonadherent cells (peripheral blood lymphocytes, PBLs) were removed. Monocytes were washed with medium and cultured for 6 days in DC culture medium consisting of RPMI1640 (Sigma) supplemented with 2 mM L-glutamine (Sigma), 50 $\mu \mathrm{M}$ 2-mercaptoethanol (Sigma), 1\% nonessential amino acids (Gibco) and $10 \%$ fetal calf serum in the presence of $20 \mathrm{ng} / \mathrm{ml} \mathrm{GM}$ CSF and IL-4 (PeproTech, London, UK). On days 3 and 5, one half of the volume of medium was replaced with fresh medium supplemented with GM-CSF and IL-4. On day 6, MDDCs were purified by positive selection using a $\mathrm{CD} 1 \mathrm{c}^{+}(\mathrm{BDCA}-1) \mathrm{DC}$ isolation kit (Miltenyi Biotec Inc., Auburn, Calif., USA).

Gag-Virus-Like Particles Activate

Dendritic Cells and NK Cells

\section{MDDC Activation and Analysis}

Human MDDCs $\left(1 \times 10^{6}\right)$ were incubated with Gag-VLPs $(10$ $\mu \mathrm{g} / \mathrm{ml}$ ), lipopolysaccharide (LPS; $1 \mu \mathrm{g} / \mathrm{ml}$; Sigma) or medium alone (control) for $24 \mathrm{~h}$. For phenotypic analysis, MDDCs were washed and stained with FITC-conjugated anti-human CD1a and PE-conjugated anti-human CD80, CD86, HLA-ABC or HLA-DR (eBioscience) and analyzed using a FACSCalibur flow cytometer and CellQuest software. Culture supernatants were collected to quantify IL-12 p70, IL-15, TNF- $\alpha$, IFN- $\alpha$ and IFN- $\gamma$ levels using ELISA kits (BD Biosciences, San Diego, Calif., USA). MDDC proliferation was evaluated using a CellTiter 96 proliferation assay kit (Promega) and carboxyfluorescein succinimidyl ester (CFSE; Invitrogen) labeling. For the proliferation assay, MDDCs treated with Gag-VLPs, LPS or medium alone were incubated in a 96-well plate $\left(5 \times 10^{3}\right.$ cells/well in $\left.100 \mu \mathrm{l}\right)$ for 5 days in triplicate wells. CellTiter 96 reagent containing MTS $(20 \mu \mathrm{l})$ was added to each well, and samples were incubated for $4 \mathrm{~h}$ at $37^{\circ} \mathrm{C}$. The absorbance of light released from formazin was recorded with a plate reader. For CFSE labeling, MDDCs $\left(5 \times 10^{5}\right)$ were loaded with $5 \mu \mathrm{M}$ CFSE and cultured for 5 days. Proliferation was analyzed by measuring the decrease in CFSE fluorescence intensity by flow cytometry after staining cells with anti-CD1a-PE.

\section{NK/DC Coculture and Analysis of NK Cell Activation}

MDDCs were treated with Gag-VLPs, LPS or medium for $24 \mathrm{~h}$ and washed thoroughly with PBS before coculturing with NK cells. Autologous human NK cells were isolated from PBLs using a $\mathrm{CD} 56^{+} / \mathrm{CD} 16^{+} \mathrm{NK}$ cell isolation kit (Miltenyi Biotec) and cultured for $12 \mathrm{~h}$ in the presence of IL-2. NK cells $\left(5 \times 10^{5}\right)$ were cocultured with MDDCs $\left(1 \times 10^{5}\right)$ at a ratio of $5: 1$ for $18 \mathrm{~h}$. NK cell activation was analyzed by flow cytometry after staining cells with anti-CD56-PE and anti-CD69-FITC.

\section{Intracellular Cytokine Staining}

Intracellular cytokine staining for IFN- $\gamma$-producing NK cells from NK/DC cocultures was performed after $18 \mathrm{~h}$ of coculture. The transport inhibitor brefeldin A $(10 \mu \mathrm{g} / \mathrm{ml}$; Sigma-Aldrich, St. Louis, Mo., USA) was added to cocultures for the last $4 \mathrm{~h}$. NK cells were surface stained with anti-CD56-PE and permeabilized with Cytofix/Cytoperm reagent (BD Pharmingen) according to the manufacturer's instructions. Cells were then stained with antiIFN- $\gamma$-FITC and examined by FACS analysis.

\section{NK Cell Proliferation}

NK cell proliferation was analyzed using the CellTiter 96 proliferation assay and CFSE labeling. NK cells were cocultured with MDDCs treated with Gag-VLP or LPS or with untreated control MDDCs at a DC to NK ratio of 1:5 for 5 days. NK cell proliferation was analyzed using the CellTiter 96 proliferation assay kit (Promega) as described above. For CFSE labeling, NK cells were labeled with $5 \mu \mathrm{M}$ CFSE and cocultured with MDDCs at a DC to NK ratio of 1:5 for 5 days. To assess the degree of proliferation, CFSE-labeled NK cells were then stained with anti-CD56-PE, and the decrease in CFSE fluorescence intensity was measured by flow cytometry.

NK Cell Cytotoxicity Assays

Autologous $\mathrm{CD}^{+} \mathrm{T}$ cells were separated from PBLs using a human $\mathrm{CD}^{+} \mathrm{T}$ cell isolation kit (Miltenyi Biotec) and cultured for $12 \mathrm{~h}$ in the presence of IL-2. CD4 ${ }^{+} \mathrm{T}$ cells were infected with 
HIV-1 (NL4-3; $100 \mathrm{ng}$ of p24 for $1 \times 10^{6}$ cells) for $2 \mathrm{~h} . \mathrm{CD}^{+} \mathrm{T}$ cells or YAC-1 cells were seeded into a 96-well round-bottomed tissue culture plate in triplicate $\left(1 \times 10^{4}\right.$ cells/well $)$ and used as target cells. Effector NK cells were cocultured with Gag-VLP or LPS-treated MDDCs for $24 \mathrm{~h}$ and added to target cells at effector to target ratios of 10:1, 5:1, 2.5:1 and 1.25:1, in a total volume of $100 \mu \mathrm{l}$, and incubated for $4 \mathrm{~h}$. Unstimulated NK cells were added as a control. Lysis of target cells was determined by measuring lactate dehydrogenase (LDH) release using the Cytotox 96 nonradioactive cytotoxicity assay kit (Promega Corp., Madison, Wisc., USA). Spontaneous release of LDH from target cells was less than $15 \%$ of the maximum release.

Immunization of Mice and Analysis of Immune Responses Six-week-old female BALB/c mice were immunized using a 2-phase immunization procedure. In the phase I immunization, 4 groups of BALB/c mice, each consisting of 3 subgroups with 4 mice each, were injected intramuscularly with $50 \mu \mathrm{g}$ of Gag-VLPs or $1 \mu \mathrm{g}$ of LPS resuspended in $100 \mu \mathrm{l}$ of sterile PBS without addition of adjuvants. Control mice were left untreated. Twenty-four hours after priming, mice from the first group were sacrificed, spleens were removed and activation of splenic DC and NK cells was assessed by flow cytometry. Seven days after priming, the first booster immunization was administered to the remaining 3 groups of mice using the same doses. Twenty-four hours after the first booster injection, mice from the second group were sacrificed and spleens were removed for flow cytometry. On day 14 , the remaining 2 groups of mice were given a second booster injection using the same doses. After $24 \mathrm{~h}$, mice from the third group (donor mice) were sacrificed, and blood samples were collected for ELISA quantification of serum IFN- $\gamma$. Spleens were removed for flow cytometry. At the same time, splenic DCs were separated from the remaining spleen cells using a mouse $\mathrm{CD} 11 \mathrm{c}^{+} \mathrm{DC}$ isolation kit (Miltenyi Biotec) and subsequently used in phase II immunizations. Mice from the fourth group were sacrificed 3 weeks after the second booster injection, and spleens were collected. A portion of the spleen cells was used for intracellular staining of IFN- $\gamma$-producing NK cells without re-stimulation. The remaining spleen cells were re-stimulated overnight with $5 \mu \mathrm{g} / \mathrm{ml} \mathrm{Gag-}$ VLPs and used for ex vivo cytotoxicity assays and intracellular staining of IFN- $\gamma$-producing NK cells. For intracellular staining, brefeldin A $(10 \mu \mathrm{g} / \mathrm{ml})$ was added, and samples were incubated for an additional $4 \mathrm{~h}$ and processed as described above.

In the phase II immunization, 4 groups of (recipient) BALB/c mice, each consisting of 5 animals, were intraperitoneally injected with purified splenic DCs $\left(1 \times 10^{6}\right.$ cells/mouse $)$ from each group of mice from the phase I immunization. All mice were sacrificed after $24 \mathrm{~h}$, serum samples were collected for the quantification of IFN- $\gamma$ and spleens were removed for flow cytometry analysis. All immunizations were performed in 2 independent experiments in accordance with institutional animal experiment guidelines.

\section{Ex vivo Cytotoxicity Assay}

NK cells were separated from the spleens of the fourth group of phase I-immunized or control mice using a mouse NK cell isolation kit (Miltenyi Biotec) after restimulation with Gag-VLPs and used as effector cells. Spleen cells isolated from non-immunized BALB/c mice were used as target cells. Target spleen cells were pulsed with Gag-VLPs $(5 \mu \mathrm{g} / \mathrm{ml})$ for $2 \mathrm{~h}$ and seeded in a 96well round-bottomed tissue culture plate $\left(1 \times 10^{4}\right.$ cells/well $)$ in triplicate. Effector NK cells were then added at effector to target ratios of 10:1, 5:1, 2.5:1 and 1.25:1 and incubated for $4 \mathrm{~h}$. Lysis of target cells was determined by measuring LDH release using the Cytotox 96 nonradioactive cytotoxicity assay kit as mentioned above.

\section{Statistical Analysis}

All data are presented as means $\pm \mathrm{SD}$. Statistical analysis was performed using Student's $t$ test. A $p$ value of $<0.05$ was considered a significant difference.

\section{Results}

Gag-VLPs Induce Activation and Proliferation of DCs

Given the crucial role of DCs in antiviral innate immunity, we sought to investigate whether HIV-1 GagVLPs could activate human DCs in vitro. Treatment of MDDCs with Gag-VLPs resulted in increased expression of the activation markers CD80, CD86, MHC I and MHC II. MHC II expression was especially elevated and reached levels higher than those observed upon treatment of MDDCs with LPS (fig. 1a). We next examined the secretion of IL-12 p70, IL-15, TNF- $\alpha$, IFN- $\alpha$ and IFN- $\gamma$ by VLP-DCs in the culture supernatant. The levels of cytokines induced by VLPs were comparable to those induced by LPS (fig. 1b). We then investigated the proliferation of MDDCs using a proliferation assay kit and CFSE labeling. The results of both experiments showed that VLPDCs and LPS-stimulated DCs (LPS-DCs) proliferated to a similar extent (fig. 1c). These results indicate that GagVLPs induce activation and maturation of DCs, which are associated with the production of specific cytokines and proliferation in vitro.

\section{Activation of NK Cells by VLP-DCs}

To determine whether human NK cells were functionally modified by VLP-DCs in vitro, MDDCs were cocultured with autologous NK cells at a 1:5 ratio for $18 \mathrm{~h}$, and NK cell activation was evaluated by measuring the expression of the NK cell activation marker CD69. NK cells cocultured with VLP-DCs exhibited a statistically significant 6-fold increase in CD69 expression over NK cells cocultured with control DCs (fig. 2a). We next assessed the amount of IFN- $\gamma$ in cocultured supernatants by ELISA. NK cells cocultured with VLP-DCs produced higher levels of IFN- $\gamma$ than did NK cells cocultured with control DCs (fig. 2b, left panel). This finding was further substantiated by intracellular staining of NK cells, which showed an increase in the frequency of IFN- $\gamma$-producing NK cells following coculture with VLP-DCs (fig. $2 b$, right panel). 


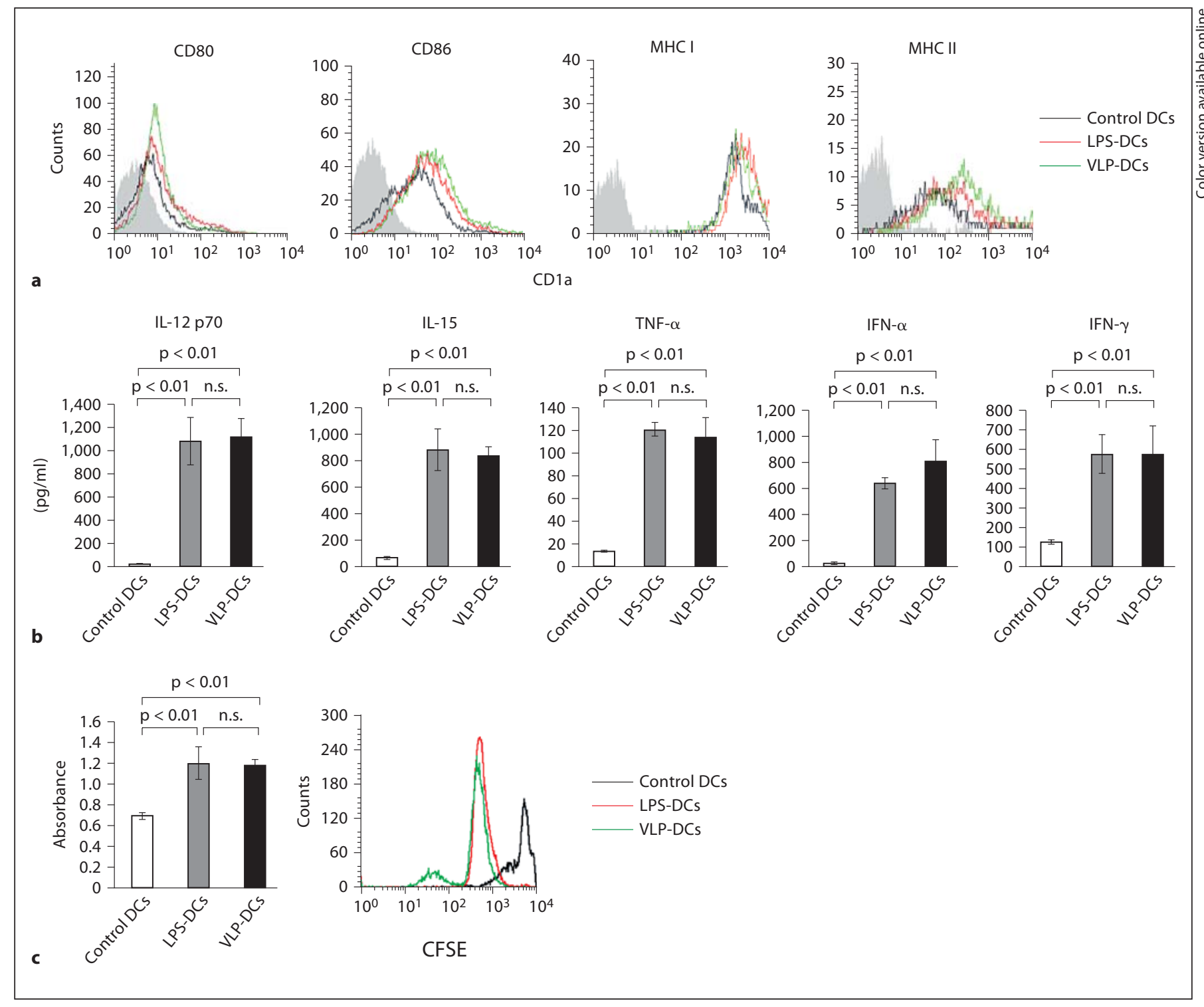

Fig. 1. Gag-VLPs induce activation and proliferation of MDDCs. a Expression of the costimulatory molecules CD80, CD86, MHC I and MHC II on human MDDCs treated with Gag-VLPs $(10 \mu \mathrm{g} /$ $\mathrm{ml})$, LPS $(1 \mu \mathrm{g} / \mathrm{ml})$ or medium alone for $24 \mathrm{~h}$ was analyzed by flow cytometry. Gray curves represent isotype controls. Results are representative of 3 independent experiments using cells from 3 different donors. b Levels of the cytokines IL-12 p70, IL-15, TNF$\alpha$, IFN- $\alpha$ and IFN- $\gamma$ in culture supernatants were measured by ELISA. All results are expressed as means \pm SD of triplicate wells. Statistical analysis was performed using Student's t test. c MDDCs

We next examined NK cell proliferation after coculture with VLP-DCs. The results of both proliferation assays (fig. 2c, left panel) and CFSE labeling of NK cells (fig. 2c, right panel) demonstrated that NK cells cocultured with VLP-DCs proliferated at a rate comparable to were treated with Gag-VLPs, LPS or medium and cultured for 5 days. MDDC proliferation was evaluated using a proliferation assay kit. The results are expressed as means \pm SD of triplicate wells. For CFSE labeling, MDDCs were labeled with $5 \mu \mathrm{M}$ CFSE and treated with Gag-VLPs, LPS or medium and cultured for 5 days. MDDC proliferation was evaluated by measuring the decrease in CFSE fluorescence intensity by flow cytometry. The results are representative of 3 independent experiments from 3 different donors.

that of NK cells cocultured with LPS-DCs after 5 days, while NK cells cocultured with control DCs showed only a basal level of proliferation. To investigate NK cell cytotoxicity, we used NK-sensitive YAC-1 cells (fig. 2d, left panel) and HIV-infected autologous $\mathrm{CD} 4^{+} \mathrm{T}$ cells (fig. $2 \mathrm{~d}$, 
right panel) as target cells and NK cells cocultured with VLP-DCs, LPS-DCs or control DCs as effector cells in cytotoxicity assays. Both the non-specific and the Gagspecific cytolytic activity of NK cells were shown to be strongest in NK cells cocultured with VLP-DCs (fig. 2d).

\section{In vivo Activation of DC and NK Cells by Gag-VLPs}

To investigate whether Gag-VLPs can induce immune responses in vivo, we introduced a 2-phase immunization strategy in $\mathrm{BALB} / \mathrm{c}$ mice as illustrated in figure $3 \mathrm{a}$. In the phase I immunization, we assessed the efficacy of Gag-VLPs in activating murine DCs and NK cells in vivo. To examine whether booster immunizations of mice with Gag-VLPs may be required to induce stronger NK cell activation and Gag-specific memory responses in vivo, we immunized 4 groups of mice and evaluated NK cell responses after each immunization.

Splenic DCs of Gag-VLP-immunized mice expressed higher levels of MHC I, MHC II and the DC activation markers CD80 and CD86 than splenic DCs of control mice, and the degree to which these markers were upregulated was similar to that observed in splenic DCs of LPS-immunized mice (fig. 3b). In vivo activation of NK cells was examined by analyzing CD69 expression on splenic NK cells after each immunization. NK cells from Gag-VLP-immunized mice showed a significant increase in the expression of CD69 after the second booster injection (fig. 3c). We next investigated serum IFN- $\gamma$ levels $24 \mathrm{~h}$ after the second booster injection (fig. 3d, left panel) and the frequency of IFN- $\gamma$-secreting splenic NK cells after restimulation with Gag-VLPs, 3 weeks after the second booster injection. Only immunization with VLPs resulted in a Gag-specific memory response, as evidenced by an increased frequency of IFN- $\gamma$-producing splenic NK cells only in VLP-immunized mice (fig. $3 \mathrm{~d}$, right panel).

Next, to investigate whether Gag-VLPs could induce Gag-specific NK cell cytolytic responses, splenic NK cells (effector cells) of the fourth group of phase I-immunized mice or control mice were restimulated and cocultured with Gag-VLP-loaded splenocytes (target cells) at various effector to target ratios, and cytotoxicity was analyzed by measuring the lysis of target cells. NK cells from Gag-VLP-immunized mice displayed a dose-dependent increase in Gag-specific cytolytic activity higher than that observed in control mice and LPS-immunized mice (fig. 3e). Together, these data demonstrate that HIV-1 Gag-VLPs induce not only NK cell innate immune responses but also Gag-specific memory responses in vivo.

\section{DCs from Gag-VLP-Immunized Mice Trigger NK}

Cell Activation and IFN- $\gamma$ Production in vivo

One of the major aims of this study was to investigate whether activation of DCs and NK cell immune responses could be induced by Gag-VLPs. Remarkably, immunization of mice with splenic DCs isolated from phase I, Gag-VLP-immunized mice resulted in NK cell activation in vivo to an extent nearly as great as that observed in mice immunized with DCs isolated from LPS-immunized mice. In contrast, NK cells of mice immunized with DCs isolated from control mice showed no increase in expression of CD69 (fig. 4a). Furthermore, ELISAs (fig. 4b, upper panel) and intracellular staining (fig. $4 \mathrm{~b}$, lower panel) demonstrated that inoculation of mice with DCs isolated from VLP- or LPS-immunized mice resulted in increased IFN- $\gamma$ secretion in vivo.

Fig. 2. Activation of human NK cells cocultured with VLP-DCs. MDDCs were treated with Gag-VLPs, LPS or medium for $24 \mathrm{~h}$. Purified autologous NK cells were added at a DC to NK ratio of 1:5 and incubated for $18 \mathrm{~h}$. a NK cell activation was analyzed by flow cytometry after staining with anti-CD56-PE and anti-CD69FITC. b Production of IFN- $\gamma$ was detected by ELISA (left panel) and intracellular cytokine staining (right panel). IFN- $\gamma$ in culture supernatants was measured using an ELISA kit. All results are expressed as means \pm SD of triplicate wells. Statistical analysis was performed using Student's t test. For intracellular cytokine staining, NK cells were stained with anti-CD56-PE, permeabilized and stained with anti-IFN- $\gamma$-FITC. IFN- $\gamma$-producing NK cells were examined by FACS analysis. The results are representative of 3 independent experiments. c NK cell proliferation was analyzed using a lymphocyte proliferation assay kit (left panel) and CFSE labeling (right panel). NK cells were cocultured with MDDCs for 5 days, and NK cell proliferation was evaluated using a proliferation assay kit. The results are expressed as means \pm SD of triplicate cultures from 3 independent experiments. For CFSE labeling, NK cells were loaded with $5 \mu \mathrm{M}$ CFSE and cocultured with MDDCs for 5 days. Proliferation was assessed by measuring the decrease in the intensity of CFSE fluorescence by flow cytometry after staining cells with anti-CD56-PE. The results are representative of 3 independent experiments using cells from 3 different donors. d To examine nonspecific and Gag-specific cytotoxicity, NK cells were cocultured with MDDCs for $24 \mathrm{~h}$. YAC-1 cells (left panel) and HIV-1 NL4-3-infected CD4 ${ }^{+}$T cells (right panel) were then added to each well $\left(1 \times 10^{4}\right.$ cells/well $)$, and samples were incubated for another $4 \mathrm{~h}$. As a control, unstimulated NK cells were incubated with target cells. Lysis of target cells was determined by measuring LDH release using the Cytotox 96 nonradioactive cytotoxicity assay kit. The results are expressed as means $\pm \mathrm{SD}$ of triplicate cultures. Comparable results were obtained in 2 independent experiments. E:T = Effector to target. 


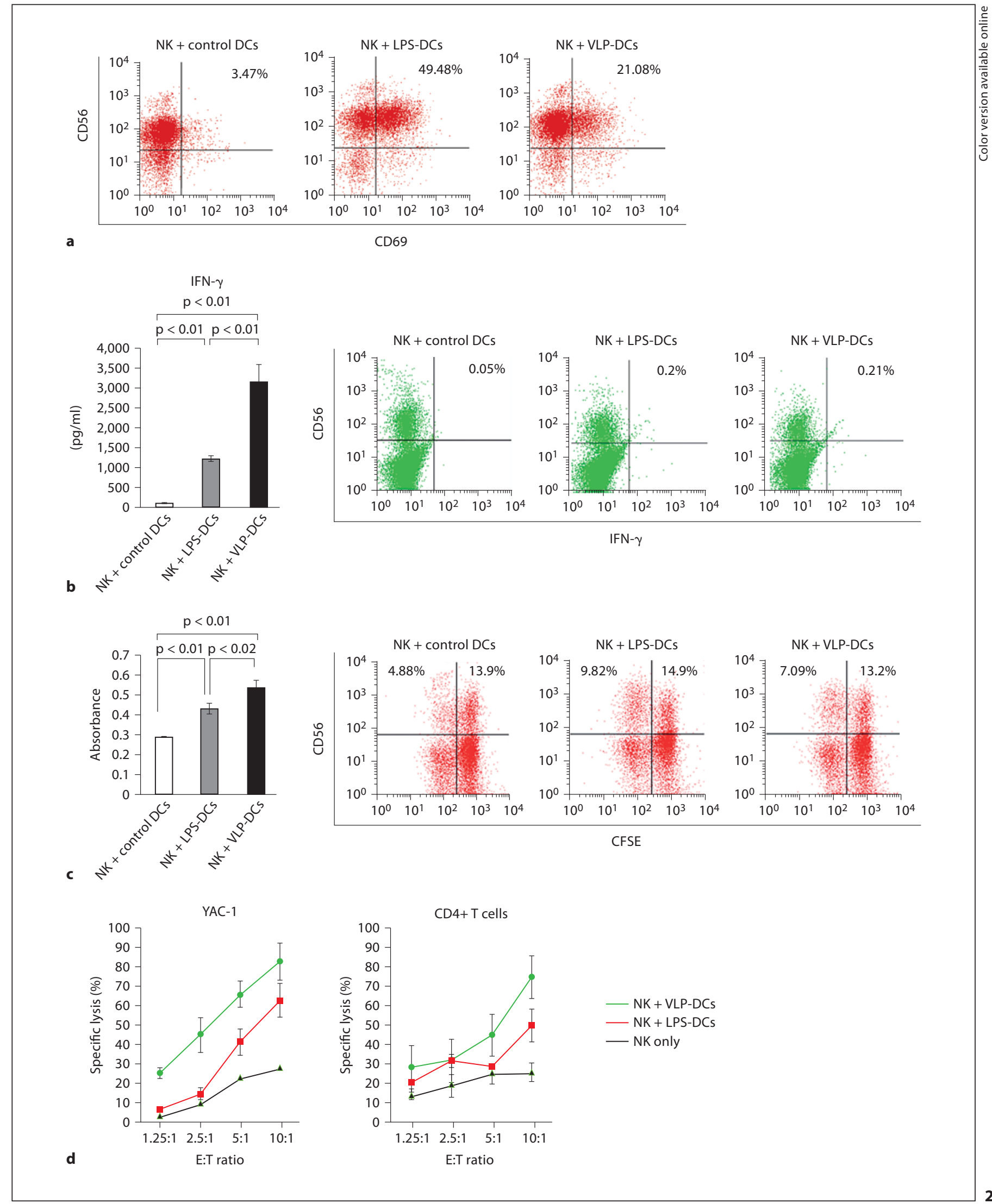


In conclusion, we show that Gag-VLPs efficiently activate human MDDCs in vitro and murine DCs in vivo. Furthermore, VLP-activated DCs subsequently induce innate immune responses as well as HIV-1-Gag-specific immune responses in NK cells.

\section{Discussion}

Despite extensive efforts to combat AIDS, the global HIV-infected population continues to increase on a daily basis. Highly active antiretroviral therapy results in significant suppression of HIV viral load but is unable to eradicate the virus from the body because of the appearance of resistant strains. One of the greatest hopes for preventing HIV infection and progression to AIDS is the development of an effective prophylactic or therapeutic vaccine.

In recent years, Gag-VLPs have been shown to be highly attractive HIV vaccine candidates because of their ability to activate multiple cell types such as DCs, T cells and B cells $[9,15,16]$. In this report, we used an HIV-1 Gag-VLP as an immunogen in a vaccine against HIV to induce DC-mediated NK cell immune responses. Previous reports showed that HIV envelope glycoprotein 120 (Env-gp120)-containing VLPs mainly induced Th2-polarizing cytokines (IL-6, IL-10 and TNF- $\alpha$ ) and failed to induce significant levels of Th1-polarizing cytokines (IL12 p70 and IFN- $\gamma$ ). The high levels of IL-10 and TNF- $\alpha$ induced by Env-VLPs could explain the aberrant function of immature DCs and impairment of T cell proliferation [32]. Moreover, gp120 induces production of IL-4, which drives synthesis of $\operatorname{IgE}$ and inhibits synthesis of antiviral IgG by B cells, inactivation of Th1 cells and inhibition of CTL responses [33]. The results from phase III trials showed that purified recombinant Env-gp120 failed to protect against HIV-1 infection and also failed to induce production of neutralizing antibodies to diverse primary isolates. The presence of HIV-1 Env-gp120 in GagVLPs switches the Th2 polarization in peripheral blood mononuclear cells from HIV-infected subjects [34-36]. For these reasons, HIV-1 Gag-VLPs are preferred as an HIV-1 vaccine candidate.

Internalization of VLPs into DCs occurs through actin-dependent macropinocytosis and endocytosis. VLP uptake is also induced by DC-SIGN-mediated endocytosis. The ability of Gag-VLPs to target DCs is an important advantage over other HIV vaccines because activation of DCs is essential for the induction of subsequent innate and adaptive immune responses. Although numerous re- ports have demonstrated Gag-VLP-mediated induction of $\mathrm{CD} 4^{+} \mathrm{T}$ cell activation, CTL responses and B-cell-mediated humoral immunity, reports on the induction of NK cell immune responses by Gag-VLPs are virtually absent.

Recent reports indicate that long-term non-progressing HIV-1 infection does not necessarily require the presence of broadly cross-reactive neutralizing antibodies; however, a strong antiviral cytotoxic activity has been shown to correlate temporally with the clearance of viremia during primary infection [37]. In contrast, reports showing a negative correlation between the number of Gag-specific $\mathrm{CD}^{+}$ T cells and HIV viral load or progression to AIDS made it necessary to reevaluate the efficiency of HIV vaccines [38]. Recently, it became apparent that a $\mathrm{CD}^{+} \mathrm{T}$ cell-inducing vaccine failed in a phase IIB clinical trial. The trial was suspended after an interim analysis showed that the vaccine did not protect the trial participants against HIV infection and indicated that the presence of high numbers of cytokine-producing HIV-specific $\mathrm{CD}^{+} \mathrm{T}$ cells does not guarantee a better clinical outcome [39]. Taken together,

Fig. 3. Immunization of mice with Gag-VLPs and assessment of DC and NK cell activation. a Schematic representation of the phase I and phase II immunization regimens. In phase I immunizations, 4 groups of female BALB/c mice, each consisting of 3 subgroups with 4 animals, were immunized by intramuscular (i.m.) injection of $50 \mu \mathrm{g}$ of Gag-VLPs or $1 \mu \mathrm{g}$ of LPS or were left untreated. Twenty-four hours after priming, mice from the first group were sacrificed and their spleens were collected for analysis of DC and NK cell activation. The remaining groups of mice were given first booster injections using the same doses 7 days after priming. Mice from the second group were sacrificed after $24 \mathrm{~h}$ and their spleens were collected. Mice from the third and fourth groups were given a second booster injection on day 14. After $24 \mathrm{~h}$, mice from the third group (donor mice) were sacrificed, and blood samples were collected for the quantification of serum IFN$\gamma$ by ELISA. Spleens were removed for flow cytometry analysis. At the same time, splenic DCs were purified using a mouse CD11 $\mathrm{c}^{+}$ DC isolation kit (Miltenyi Biotec) and subsequently used in phase II immunizations. Mice from the fourth group were sacrificed 3 weeks after the second booster injection, and spleens were collected for flow cytometry. A portion of splenocytes was restimulated overnight with $5 \mu \mathrm{g} / \mathrm{ml} \mathrm{Gag-VLPs}$ and used for intracellular staining of IFN- $\gamma$-producing NK cells and ex vivo cytotoxicity assays. In phase II immunizations, 4 groups of (recipient) BALB/c mice, each consisting of 5 animals, were adoptively transferred with purified splenic DCs $\left(1 \times 10^{6}\right.$ cells/mouse $)$ from phase Iimmunized (donor) mice from the third group. After $24 \mathrm{~h}$, all mice were sacrificed and sera and spleens were collected for immunological assays. b Expression of mouse CD80, CD86 and MHC I and MHC II molecules on splenic DCs from the first group of phase I-immunized mice was analyzed by flow cytometry. 


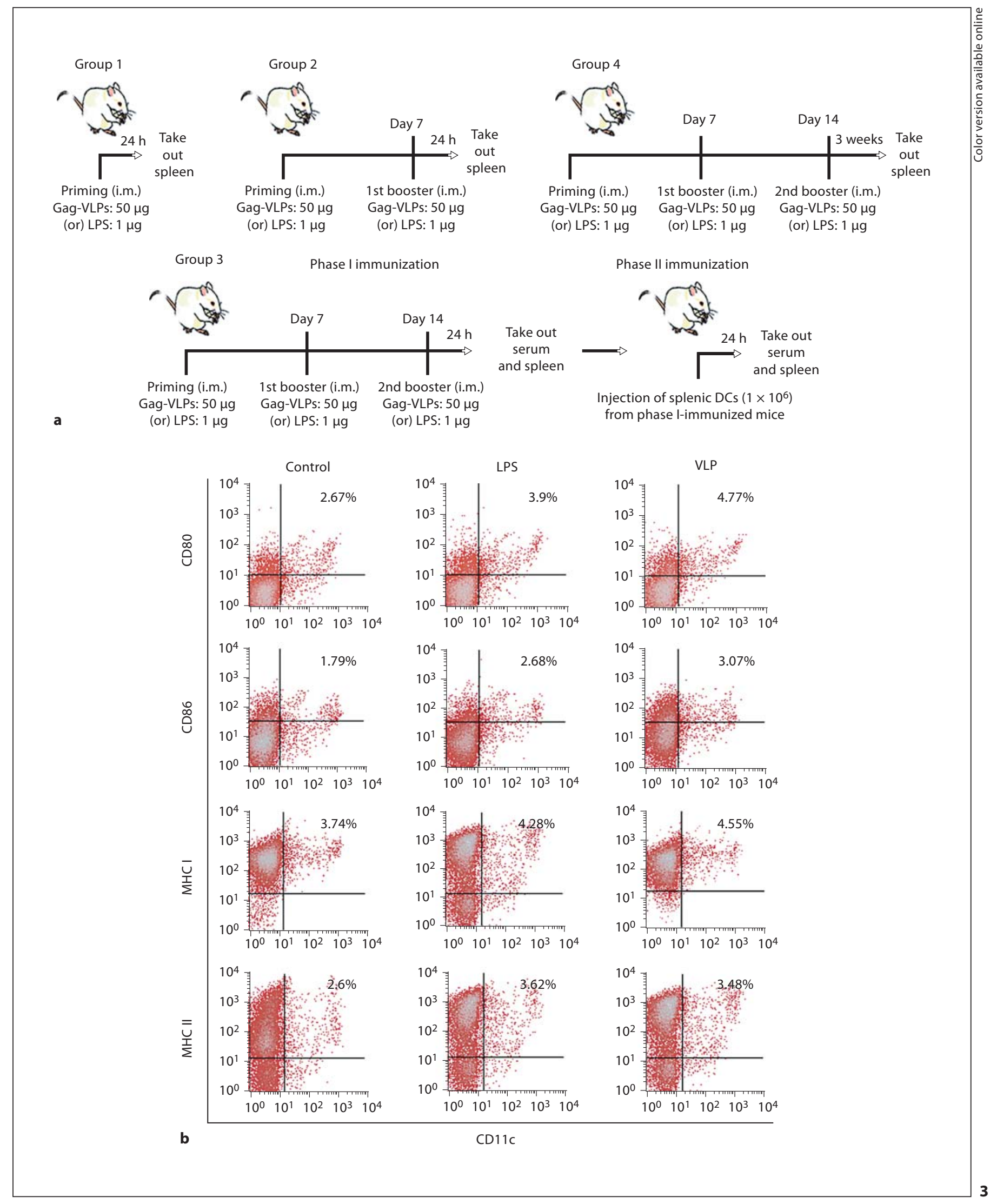




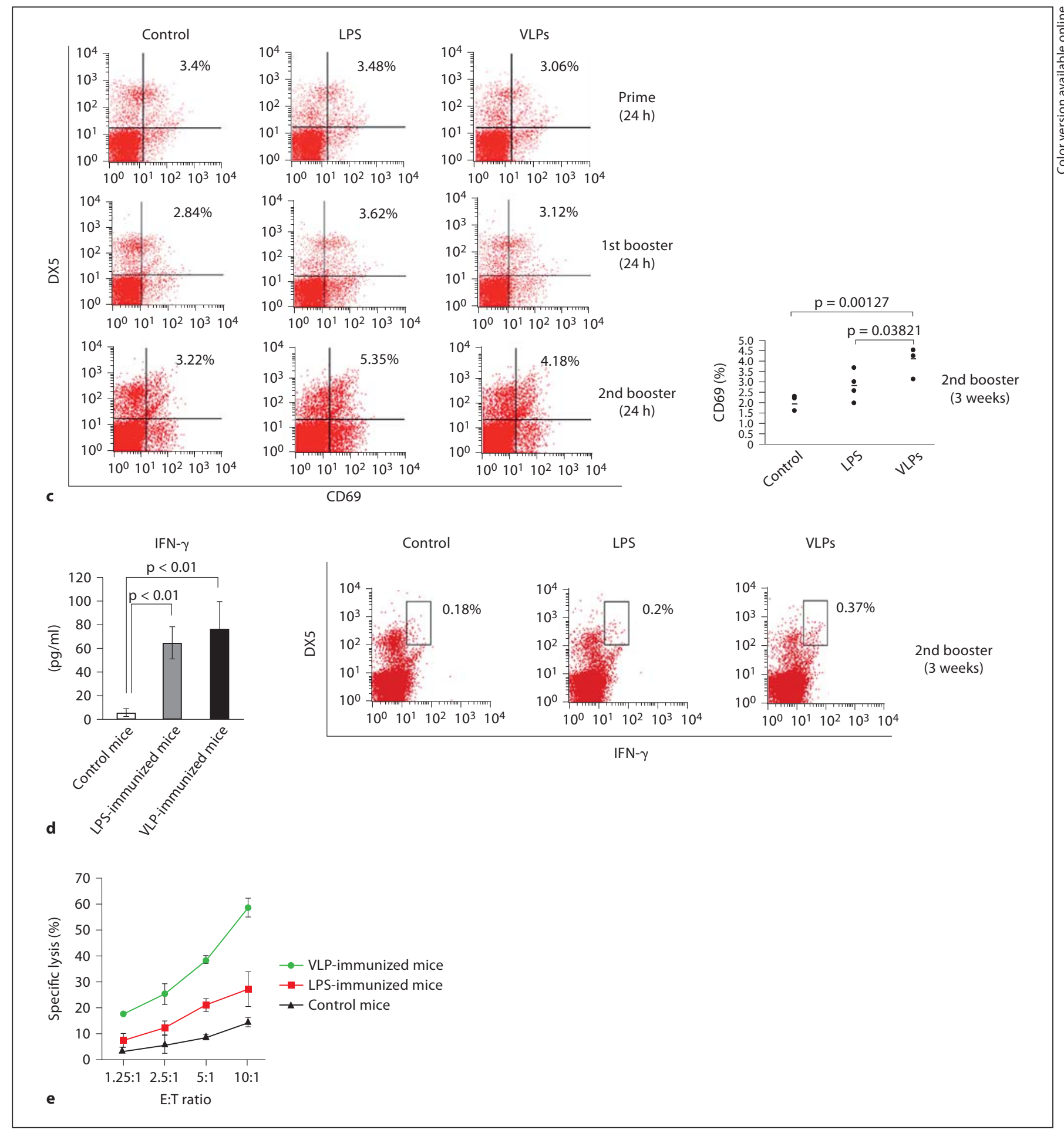

Fig. 3. Immunization of mice with Gag-VLPs and assessment of DC and NK cell activation. c CD69 expression on NK cells from each group of phase I-immunized mice was analyzed by flow cytometry. d Serum IFN- $\gamma$ levels from the third group of phase Iimmunized mice were determined by ELISA. IFN- $\gamma$-producing splenic NK cells from the fourth group of phase I-immunized mice after restimulation with Gag-VLPs were examined by intra- cellular cytokine staining. The results are representative of 3 independent experiments. e Gag-specific NK cell cytolysis of GagVLP-loaded spleen cells was assessed by measuring LDH release using the Cytotox 96 non-radioactive cytotoxicity assay kit. The results are expressed as means $\pm \mathrm{SD}$ of triplicate cultures. Comparable results were obtained in 2 independent experiments. $\mathrm{E}: \mathrm{T}=$ Effector to target. 


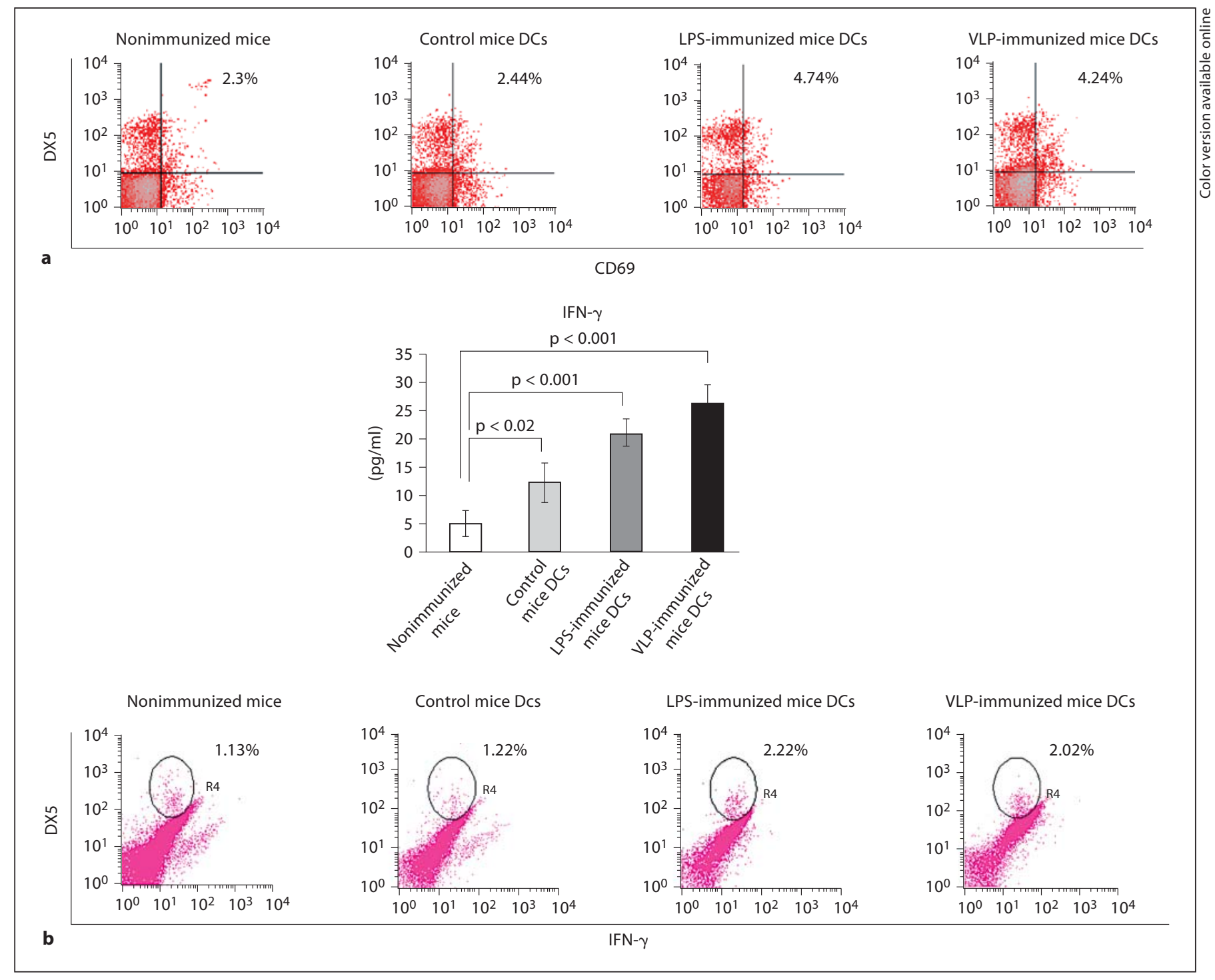

Fig. 4. DCs from phase I-immunized donor mice induce in vivo activation of NK cells. a NK cell activation in phase II, DC-immunized recipient mice was analyzed by flow cytometry after staining the spleen cells with antibodies against DX5-PE and antiCD69-FITC. b Serum IFN- $\gamma$ levels of phase II-immunized or control mice were determined using a mouse IFN- $\gamma$ ELISA assay kit.

these results suggest that in order to achieve an effective potential HIV vaccine, a strategy that results in induction of both nonspecific innate immune responses and specific immune responses against HIV is necessary.

In this study, we first demonstrated the activation of DCs and NK cells by Gag-VLPs both in vitro and in vivo by immunization with Gag-VLPs or splenic DCs isolated from VLP-immunized mice in a 2-phase immunization experiment.
All results are expressed as means \pm SD of triplicate wells. Statistical analysis was performed using Student's t test. IFN- $\gamma$-producing murine NK cells were examined by intracellular cytokine staining and FACS analysis. The results are representative of 3 independent experiments.

We generated HIV-1 Gag-VLPs in mammalian HeLa cells to exclude the possible baculovirus contamination of VLP preparations, which may have affected the results of experiments intended to analyze the immunogenicity of VLPs. Our studies using these VLP preparations showed that incubation of human MDDCs with GagVLPs resulted in upregulation of the DC maturation markers MHC I and MHC II and of costimulatory molecules such as CD80 and CD86 (fig. 1a). DC activation is

J Innate Immun 2012;4:187-200 
also characterized by the production of cytokines that are important for the priming of innate and adaptive immune cells such as NK cells, $\mathrm{CD} 4^{+}$and $\mathrm{CD} 8^{+} \mathrm{T}$ cells. Thus, to determine the type of responses elicited by DCs stimulated with VLPs, we examined the secretion of IL12 p70, IL-15, TNF- $\alpha$, IFN- $\alpha$ and IFN- $\gamma$ in culture supernatants and demonstrated that Gag-VLP-treated DCs produced increased levels of cytokines (fig. 1b). The quality and the quantity of DCs are important factors when attempting to induce effective immune responses in T cells and NK cells. To be effective, a vaccine must restore both the function and the number of DCs in HIVinfected persons. In vivo expansion of DCs after immunization has been shown to be necessary for the activation of other immune cells [16]. Moreover, since DC immunotherapy requires a sufficient quantity of functional autologous DCs, it is important that stimulation with Gag-VLPs should induce the activation and maturation of DCs as well as proliferation of functional DCs. We next used 2 methods to investigate the ability of GagVLPs to induce proliferation of human DCs in vitro. The results of CFSE labeling experiments and experiments using a proliferation assay kit both demonstrated the proliferation of VLP-treated MDDCs (fig. 1c). To further prove the capability of VLP-treated DCs to stimulate NK cells, coculture experiments were performed to analyze NK cell immune responses. These experiments demonstrated that NK cells cocultured with VLP-treated DCs showed upregulation of CD69 expression, IFN- $\gamma$ production, proliferation and cytotoxicity against YAC-1 cells and HIV-infected CD4+ T cells (fig. 2). Furthermore, MDDCs produced IFN- $\gamma$ after stimulation with Gag-VLPs or LPS, but the presence of NK cells in cocultures significantly increased IFN- $\gamma$ levels (about 8-fold). Because the number of DCs in cocultures was 10 times less than that in DC-only cultures, we hypothesized that the IFN- $\gamma$ in NK/DC cocultures was produced mainly by NK cells.

Recent reports have demonstrated NK cell memory for specific antigens and NK cell involvement in both innate and adaptive immunity. Rapid NK cell responses during innate immune activation are also associated with longlasting antigen-specific NK cell memory responses [4044]. NK cells can develop memory-like properties based on prior activation. These amplified NK cell responses are important for the early response to pathogens, and it may be possible to boost the NK cell response to subsequent infection by stimuli that result in the memory-like NK cell phenotype. NK cells can orchestrate specific immune responses to infection by recognizing pathogens through germ-line-encoded receptors such as toll-like receptors [45].

In our studies, immunization of mice with Gag-VLPs resulted in activation of murine DCs and NK cells in vivo, supporting the results of in vitro experiments (fig. 3b-e). We evaluated NK cell activation $24 \mathrm{~h}$ after each immunization with Gag-VLPs (prime, first and second booster injections) to verify the immediate NK cell response to each immunization and again 3 weeks after the last immunization to verify Gag-specific NK cell memory as shown in figure $3 \mathrm{~d}$ (right panel) and e. We found that the prime/boost regimen using Gag-VLPs significantly enhanced NK cell activation and Gag-specific NK cell immunity. In both LPS-immunized mice and Gag-VLP-immunized mice, nonspecific activation of NK cells was evidenced by the increase in CD69 expression shortly after (24 h) each immunization (fig. 3c). In contrast, long-lasting Gag-specific NK cell immune responses of NK cells were only seen in Gag-VLP-immunized mice (fig. 3d, right panel, e). These results demonstrate that Gag-VLPs are able to induce NK cell innate immune responses as well as HIV-1 Gag-specific memory responses.

Sailaja et al. [16] injected mice intraperitoneally with Gag-VLPs once and assessed the activation of splenic DCs, T cells and B cells on day 3. Our data showed that $24 \mathrm{~h}$ after priming, NK cell activation was not detected in the first group of mice. This is likely due to the early time point at which the mice were examined, a short time (24 h) after injection with Gag-VLPs, which is likely too short a time period to induce DC-mediated NK cell activation in vivo. NK cell activation was found to increase after each booster injection due to the increased activation of DCs induced by booster injections (fig. 3c).

Adoptive transfer of DCs from VLP-immunized mice in the phase II immunization experiments revealed that Gag-VLP-immunized DCs could induce NK cell innate and Gag-specific immune responses in vivo (fig. 4a, b).

In most previous adoptive transfer studies, mice were injected intraperitoneally with $1 \times 10^{6} \mathrm{DCs}$ [46]. Transferred DCs reportedly localized in the spleen as early as $3 \mathrm{~h}$ after intravenous injection and persisted for $24 \mathrm{~h}$. DCs entering the popliteal lymph nodes from the footpad were also located $3 \mathrm{~h}$ after subcutaneous transfer [47]. Eggert et al. [48] injected mice subcutaneously, intraperitoneally and intravenously with $1 \times 10^{6} \mathrm{DCs} /$ mouse. In intravenously injected mice, DCs localized in the lung $5 \mathrm{~min}$ after injection and in the spleen as early as $2 \mathrm{~h}$ after injection. DCs were detected in the draining lymph nodes $8 \mathrm{~h}$ 
after intraperitoneal injection [48]. DCs adhere to endothelium within the splenic marginal zone through endothelium-specific homing receptors and chemokine receptor expression [49]. The required number of DCs, DC trafficking/migration patterns and subsequent immune responses differ greatly according to cell type, route of inoculation and the immune competency of recipient mice.

In this study, we evaluated the ability of Gag-VLPs to induce NK cell innate responses and HIV-1 Gag-specific NK cell adaptive immunity, which is essential for the immunological control of HIV-1 infection. These studies highlighted the role of DCs in VLP-based vaccine strategies. Our findings have significant implications for the design of a highly effective vaccine model for prophylactic and therapeutic purposes in HIV infection.

\section{Acknowledgements}

We thank Mr. Yuuta Kasai and Miss Megumi Watanabe for helpful discussions as well as for their excellent work in performing immunizations and ELISA. This work was supported, in part, by a Grant-in-Aid for High Technology Research (No. 09309011) from the Ministry of Education, Science, Sports and Culture, Japan, by a Grant-in-Aid for AIDS research from the Ministry of Health, Labor and Welfare, Japan, by a grant from the Supporting Program for Creating University Ventures from the Science and Technology Agency, Japan, and by a grant for the Research and Development Program for New Bio-industry Initiatives from the Ministry of Agriculture, Forestry and Fisheries of Japan.

\section{Disclosure Statement}

The authors declare that they have no competing interests.

\section{References}

1 Murphey-Corb M: Live-attenuated HIV vaccines: how safe is safe enough? Nat Med 1997; 3:17-18.

$\checkmark 2$ Ruprecht RM: Live attenuated AIDS viruses as vaccines: promise or peril? Immunol Rev 1999;170:135-149.

-3 Robertson JS, Griffiths E: Assuring the quality, safety, and efficacy of DNA vaccines. Mol Biotechnol 2001;17:143-149.

4 Noad R, Roy P: Virus-like particles as immunogens. Trends Microbiol 2003;11:438-444.

5 Grgacic EV, Anderson DA: Virus-like particles: passport to immune recognition. Methods 2006;40:60-65.

6 Ludwig C, Wagner R: Virus-like particles universal molecular toolboxes. Curr Opin Biotechnol 2007; 18:537-545.

7 Doan LX, Li M, Chen C, Yao Q: Virus-like particles as HIV-1 vaccines. Rev Med Virol 2005; $15: 75-88$.

8 Young KR, McBurney SP, Karkhanis LU, Ross TM: Virus-like particles: designing an effective AIDS vaccine. Methods 2006; 40 : 98-117.

-9 Buonaguro L, Tornesello ML, Tagliamonte M, Gallo RC, Wang LX, Kamin-Lewis R, Abdelwahab S, Lewis GK, Buonaguro FM: Baculovirus-derived human immunodeficiency virus type 1 virus-like particles activate dendritic cells and induce ex vivo T-cell responses. J Virol 2006;80:9134-9143.

10 Tsunetsugu-Yokota Y, Morikawa Y, Isogai M, Kawana-Tachikawa A, Odawara T, Nakamura T, Grassi F, Autran B, Iwamoto A: Yeast-derived human immunodeficiency virus type 1 p55(gag) virus-like particles activate dendritic cells (DCs) and induce perforin expression in Gag-specific CD8(+) T cells by cross-presentation of DCs. J Virol 2003; 77:10250-10259.
11 Morón VG, Rueda P, Sedlik C, Leclerc C: In vivo, dendritic cells can cross-present viruslike particles using an endosome-to-cytosol pathway. J Immunol 2003; 171:2242-2250.

12 Deml L, Wild J, Wagner R: Virus-like particles: a novel tool for the induction and monitoring of both T-helper and cytotoxic Tlymphocyte activity. Methods Mol Med 2004;94:133-157.

13 Paliard X, Liu Y, Wagner R, Wolf H, Baenziger J, Walker CM: Priming of strong, broad, and long-lived HIV type 1 p55gag-specific $\mathrm{CD}^{+}$cytotoxic $\mathrm{T}$ cells after administration of a virus-like particle vaccine in rhesus macaques. AIDS Res Hum Retroviruses 2000; 16:273-282.

14 Deml L, Speth C, Dierich MP, Wolf H, Wagner R: Recombinant HIV-1 Pr55gag viruslike particles: potent stimulators of innate and acquired immune responses. Mol Immunol 2005;42:259-277.

$\longrightarrow 15$ Buonaguro L, Visciano ML, Tornesello ML, Tagliamonte M, Biryahwaho B, Buonaguro FM: Induction of systemic and mucosal cross-clade neutralizing antibodies in $\mathrm{BALB} / \mathrm{c}$ mice immunized with human immunodeficiency virus type 1 clade A viruslike particles administered by different routes of inoculation. J Virol 2005;79:70597067.

16 Sailaja G, Skountzou I, Quan FS, Compans RW, Kang SM: Human immunodeficiency virus-like particles activate multiple types of immune cells. Virology 2007;362:331-341.

-17 Speth C, Bredl S, Hagleitner M, Wild J, Dierich $\mathrm{M}$, Wolf $\mathrm{H}$, Schroeder J, Wagner $\mathrm{R}$, Deml L: Human immunodeficiency virus type-1 (HIV-1) Pr55gag virus-like particles are potent activators of human monocytes. Virology 2008;382:46-58.
18 Inaba K, Metlay JP, Crowley MT, WitmerPack M, Steinman RM: Dendritic cells as antigen presenting cells in vivo. Int Rev Immunol 1990;6:197-206.

19 Steinman RM, Hemmi H: Dendritic cells: translating innate to adaptive immunity. Curr Top Microbiol Immunol 2006;311:1758.

20 Steinman RM, Banchereau J: Taking dendritic cells into medicine. Nature 2007;449: 419-426.

21 Zitvogel L: Dendritic and natural killer cells cooperate in the control/switch of innate immunity. J Exp Med 2002;195:F9-F14.

22 Cooper MA, Fehniger TA, Fuchs A, Colonna $\mathrm{M}$, Caligiuri MA: NK cell and DC interactions. Trends Immunol 2004;25:47-52.

23 Moretta A: The dialogue between human natural killer cells and dendritic cells. Curr Opin Immunol 2005; 17:306-311.

- 24 Brilot F, Strowig T, Munz C: NK cells interactions with dendritic cells shape innate and adaptive immunity. Front Biosci 2008;13: 6443-6454.

25 Wehner R, Dietze K, Bachmann M, Schmitz M: The bidirectional crosstalk between human dendritic cells and natural killer cells. J Innate Immun 2011;3:258-263.

-26 Andoniou CE, Coudert JD, Degli-Esposti MA: Killers and beyond: NK-cell-mediated control of immune responses. Eur J Immunol 2008;38:2938-2942.

27 Brandstadter JD, Yang Y: Natural killer cell responses to viral infection. J Innate Immun 2011;3:274-279.

28 Guilmot A, Hermann E, Braud VM, Carlier Y, Truyens C: Natural killer cell responses to infections in early life. J Innate Immun 2011; 3:280-288 
-29 Kottilil S, Chun TW, Moir S, Liu S, McLaughlin M, Hallahan CW, Maldarelli F, Corey L, Fauci AS: Innate immunity in human immunodeficiency virus infection: effect of viremia on natural killer cell function. J Infect Dis 2003;187:1038-1045.

$\checkmark 30$ Fauci AS, Mavilio D, Kottilil S: NK cells in HIV infection: paradigm for protection or targets for ambush. Nat Rev Immunol 2005; 5:835-843.

-31 Osada T, Clay T, Hobeika A, Lyerly HK, Morse MA: NK cell activation by dendritic cell vaccine: a mechanism of action for clinical activity. Cancer Immunol Immunother 2006;55:1122-1131.

-32 Fantuzzi L, Purificato C, Donato K, Belardelli F, Gessani S: Human immunodeficiency virus type 1 gp120 induces abnormal maturation and functional alterations of dendritic cells: a novel mechanism for AIDS pathogenesis. J Virol 2004;78:9763-9772.

33 Becker Y: HIV-1 induced AIDS is an allergy and the allergen is the Shed gp120 - a review, hypothesis, and implications. Virus Genes 2004;28:319-331.

-34 Burton DR, Desrosiers RC, Doms RW, Koff WC, Kwong PD, Moore JP, Nabel GJ, Sodroski J, Wilson IA, Wyatt RT: HIV vaccine design and the neutralizing antibody problem. Nat Immunol 2004;5:233-236.

>35 Buonaguro L, Tornesello ML, Gallo RC, Marincola FM, Lewis GK, Buonaguro FM: Th2 polarization in peripheral blood mononuclear cells from human immunodeficiency virus (HIV)-infected subjects, as activated by HIV virus-like particles. J Virol 2009; $83: 304-313$
36 Cohen J: HIV/AIDS. Vaccine results lose significance under scrutiny. Science $2003 ; 299$. 1495.

37 Harrer T, Harrer E, Kalams SA, Elbeik T, Staprans SI, Feinberg MB, Cao Y, Ho DD, Yilma T, Caliendo AM, Johnson RP, Buchbinder SP, Walker BD: Strong cytotoxic T cell and weak neutralizing antibody response in a subset of persons with stable nonprogression HIV type I infection. AIDS Res Hum Retroviruses 1996;12:585-592.

38 Schellens IM, Borghans JA, Jansen CA, De Cuyper IM, Geskus RB, van Baarle D, Miedema F: Abundance of early functional HIVspecific $\mathrm{CD}^{+} \mathrm{T}$ cells does not predict AIDSfree survival time. PLoS One 2008;3:e2745.

39 Vaccination and enrollment are discontinued in phase II trials of Merck's investigational HIV vaccine candidate. Press release from Merck and the HIV Vaccine Trials Network, Sept 21, 2007. http://www.hvtn.org/ pdf/FINAL_HIV_Vaccine_Press_Release. pdf.

40 Cooper MA, Colonna M, Yokoyama WM: Hidden talents of natural killers: NK cells in innate and adaptive immunity. EMBO Rep 2009;10:1103-1110.

41 Cooper MA, Yokoyama WM: Memory-like responses of natural killer cells. Immunol Rev 2010;235:297-305.

42 Sun JC, Beilke JN, Lanier LL: Adaptive immune features of natural killer cells. Nature 2009;457:557-561.
43 Ugolini S, Vivier E: Immunology: natural killer cells remember. Nature 2009;457:544545.

44 Paust S, Senman B, von Andrian UH: Adaptive immune responses mediated by natural killer cells. Immunol Rev 2010;235:286-296.

45 Janeway CA Jr, Medzhitov R: Innate immune recognition. Annu Rev Immunol 2002;20: 197-216.

46 Yoshida O, Akbar F, Miyake T, Abe M, Matsuura B, Hiasa Y, Onji M: Impaired dendritic cell functions because of depletion of natural killer cells disrupt antigen-specific immune responses in mice: restoration of adaptive immunity in natural killer-depleted mice by antigen-pulsed dendritic cell. Clin Exp Immunol 2008;152:174-181.

47 Austyn JM, Kupiec-Weglinski JW, Hankins DF, Morris PJ: Migration patterns of dendritic cells in the mouse. Homing to T celldependent areas of spleen, and binding within marginal zone. J Exp Med 1988;167:646651.

48 Eggert AA, Schreurs MW, Boerman OC, Oyen WJ, de Boer AJ, Punt CJ, Figdor CG, Adema GJ: Biodistribution and vaccine efficiency of murine dendritic cells are dependent on the route of administration. Cancer Res 1999;59:3340-3345.

-49 Barratt-Boyes SM, Zimmer MI, Harshyne LA, Meyer EM, Watkins SC, Capuano S 3rd, Murphey-Corb M, Falo LD Jr, Donnenberg AD: Maturation and trafficking of monocyte-derived dendritic cells in monkeys: implications for dendritic cell-based vaccines. J Immunol 2000;164:2487-2495. 